\title{
The Development of Chemico-mechanical Practices in Polymer Material Machining by Cutting
}

\author{
Kong Lingbo ${ }^{1}$, Qin Hongwu ${ }^{2}$, Zhang $\mathrm{Xian}^{3}$ and Fan Qinyin ${ }^{4}$ \\ ${ }^{1}$ College of Machinery and Vehicle Engineering \\ ${ }^{2}$ College of Electronic and Information Engineering \\ ${ }^{3}$ International Education College \\ Changchun University \\ Changchun, China \\ ${ }^{4}$ Graduate School of Engineering \\ Osaka University, \\ Osaka, Japan \\ 568260932@qq.com ${ }^{3}$, fqyyhoo@yahoo.co.jp ${ }^{4}$
}

\begin{abstract}
The analysis of phenomena taking place when polymers interact with aggressive mediums has been made. Corresponding experimental research has been done and methods to direct changes of proceed material have been developed. The changes happen when the surface layer is processed preliminary to lower resistance to destruction of work piece surface layer when it interacts with cutting instrument later. The polymeric composites materials (PCM), having specific characteristic self lubrication, have got the name-the materials antifrictional selflubrication (MAS). In compositions MAS except matrixes-of the thermoreactive resins are included special fillers. The structure material in defines the characteristic received products. For the reason increasing working characteristic PCM necessary study of the influence of the thermodynamic conditions centrifugal litho on shaping the structure PCM. For study of the influence technological parameters is required corresponding to equipments.
\end{abstract}

Keywords: PCM, MAS, NDT, chemical medium, SAS, polymer materials

\section{Introduction}

The destruction of constructional material structures, including polymers, is effective chemical medium [1-2].the influence of the aggressive medium on polymer is expressed in changing its structure and properties without destructions of the material integrity (cracking, loosening or swelling of the material surface to a certain degree) or causing its destruction. For nondestructive testing (NDT) by acoustic methods it is often necessary to generate probe pulses of complex forms [3-4]. The effectiveness and reliability of results from this testing depends on the accuracy and ability to reproduce these pulses. This raises the problem of synthesizing such complex signals on predetermined parameters. Setting for these parameters are both time and frequency domains. The necessary signal parameters could be changed during operation, depending on the properties of the object, as well as the technical parameters of the machinery used and the properties of the transmission lines.

In this connection the analysis of the processes and effects when chemical medium is interacting with polymers is of scientific interest as well as the development on this basis chemical-mechanical method of polymeric work pieces processed by cutting. Aggressive 
mediums can be derided info physically and chemically active in accordance with their interaction with polymers. Active substances have specific influence on polymer surface active substances (SAS), they can belong to both groups. The influence of physically active mediums consists in diffusing into polymer and weakening ties between molecules which causes the lowering of strength properties of the surface layer of a material. Aggressive chemical mediums when in contact with polymeric material cause irreversible changing of their chemical structure. Totality of chemical processes causing chemical structure changes of the polymer and its molecular mass is called chemical destruction.

The influence of physically active mediums consists in diffusing into polymer and weakening ties between molecules which causes the lowering of strength properties of the surface layer of a material. Aggressive chemical mediums when in contact with polymeric material cause irreversible changing of their chemical structure. Totality of chemical processes causing chemical structure changes of the polymer and its molecular mass is called chemical destruction. When polymers of physically active mediums consists in diffusing into polymer and weakening ties between molecules which causes the lowering of strength properties of the surface layer of a material. Aggressive chemical mediums when in contact with polymeric materials cause irreversible changing of their chemical structure. Totality of chemical processes causing chemical structure changes of the polymer and its molecular mass is called chemical destruction. When polymers interact of with aggressive mediums a number of physical and chemical processes can take please the most important of them are the following: adsorption of aggressive mediums components on the polymer surface; diffusion of aggressive mediums in the polymer volume; chemical reaction in aggressive mediums with chemically polymer unstable ties of polymer; diffusion of destruction products to the polymer surface, desorption of destruction products from the surface of the polymer. The influence of physically active aggressive mediums on the stable properties of polymer materials (PM), determining the resistance to destruction, can be volumetric (swelling) and shallow (adsorption, surface diffusion). In case of adsorption only surface part of solid take part the process; the chemical potential of solid is not changing. "Surface part of solid" means not only act side surface, but inner surface around molecular groups and probably the surface of particular molecules. It is evident that the effective surface of solid depends on a configuration of its molecules and on some properties diffusing substances: the size of molecules and their forms. Adsorption will take place on a inner parts of solid surface which are formed by the walls of emptiness and pores, which have enough volume and the corresponding form collecting molecules of diffusing substance. When swelling takes place in physically active mediums the solidity of polymer material is lowering while its elasticity is improving [5-8]. The lowering of polymer solidity under swelling can be explained by two factors.

First, it can be caused by inter (local) tension as a result of irregular swelling. In the process of dissolvent sorption by polymers different areas with different degree of swelling can be observed [9]. It is characteristic for polymers in glassy condition. This phenomenon is caused by great dependence of diffusion factor on diffusing component component concentration in system as well as specific interaction of polymer with substance. In such system in sorption process because of unequal movement of molecules of substance being sorbet and because of polymer segment non-permanent tensions can appear. Sometimes these tensions are considerable. The parts of the neighboring hard part which has not been swelled and where stretching tensions take place. The irregular swelling and surface changes as will as different inner tensions lead to formation of considerable number of micro cracks and inner micro defects. Local tensions can be enough to break "SS" connections. 
Second, solidity can be changed as a result of intermolecular interaction which can be observed in the process of polymer swelling, But as it was mentioned above, swelling of a polymer material can contribute to improving the elasticity, which means improving its chain flexibility and lowering the temperature of vitrifying. The increasing of flexibility of polymer chains contributes to better orientation of macromolecules under mechanical loading, including the case of cutting forces under mechanical processing which cutting instrument. In this case an effect of deformation solidity of the material can be observed, it means material solidity in improving when it is necessary to reduce resistibility to destruction. In this connection a conclusion can be made, that in the aspect of effectiveness of the following mechanical processing of polymer materials the swelling as a result of interaction of aggressive medium and polymer is not desirable. It is necessary to do processing of polymers in the aggressive medium to ensure cracking and loosening of the surface layer not deeply without changing chemical properties of a certain polymer.

The essence of adsorption effect of the medium in the process of destruction consists of two effects - outer (energetic) and inner (mechanical). The inner absorption effect takes place when the surface active substances on the surface of defects inside solid are absorbed, It ensures difficulties in connecting cracks, deepening and increasing defect is caused by absorption of active medium $n$ the outer surface of solid which results in lowering the limit of fluidity and solidity factor[10]. The characteristics of plastic deformation; resistibility to creping, solidity, inner friction of solids depend on surface state.

The solidity of materials is in proportion to their surface energy; so factors causing reducing of free surface energy, reducing of forming new surface lowering material solidity. Reducing surface energy as a result of absorption on the boarder "solid-environment" makes the process of appearing and developing tracks of destruction easy and it is the reason of lowering solidity of the material. This phenomenon is observed when SAS is used as an aggressive medium.

\section{Experimental Data}

The influence of SAS on durability, solidity and deformability has been discovered and studied by academician P. A. Rebinder. Rebinder's effect (reducing durability under absorption of SAS) has been discovered for many solids, including polymer. It has been found out that a number of liquids (water, vinegar acid) being not good lubricants, has the ability to lower specific load of some materials. And vise versa, liquids which lower friction (ethyl alcohol) do not influence reducing specific cutting work greatly. It can be explained by absorption effect of durability lowering.

The influence of SAS leading to lowering of solidity is explained by the fact that there are cracks on the surface which appear in technological process of producing polymer material. SAS is physically absorbed on the surface getting into every defect; its quality is determined by the equation for pressure developed in capillaries:

$$
\Delta P=\frac{2 \gamma \cos \theta}{r}
$$

Where $\triangle \mathrm{P}$ - pressure across meniscus; $r$ - radius of pore; $\theta$ - the angel of contact, characterizing the degree of wetting; $\gamma$-free surface energy.

When the material is wetted fully $(\cos \theta=1), \Delta P_{\max }=2 \gamma / r$, when $r$ is small, pressure can be very high.

It is difficult to define surface of solids to value the influence of different mediums on it experimentally, so Yung's ratio is used. 


$$
\gamma_{m . \mathrm{n}}=\gamma_{m . \mathrm{z}}-\gamma_{n . \mathrm{z}} \cos \theta
$$

Where $\theta$ - the edge angle of wetting; $\gamma$-free surface energy (indexes under $\gamma$ belong to corresponding surface). For given polymer $\gamma_{m . z}$ is constant and the change $\gamma_{m . n}$ depends on the value of surface liquid tension $\gamma_{\mathrm{n} . \mathrm{z}}$ when polymer's wetted fully $(\cos \theta=1)$. In this case if $\gamma_{n . z}$ increases, $\gamma_{m . n}$ decreases and resistance of polymer to destruction must lower. When wetting is not full the increase of $\gamma_{n . z}$ when $\cos \theta$ is changing can lead to either decreasing of $\gamma_{m . n}$ or its increasing.

It has been found out, that under temperatures lower than vitrifying temperature crystal areas physically are not influenced by most of sobbing substances. Analyzing the results of experiments with different samples has allowed making a conclusion that solubility is in proportion to the part of volume of amorphous phase:

$$
S=S_{a} \varphi a
$$

Where $S_{a}$ - solubility of sobbing substance in fully amorphous polymer.

In crystal polymer the influence of dissolvent is load on the boarders of spherolits, sometimes inside spheroids between rays. It is explained by the fact in the process of crystallization in spheroids structural units of similar structure are getting in order, for example, in line polymer - line molecules.

In this case molecules containing ramifications and extraneous groups, being the result of oxidation and other process, automatically are being pushed off from crystals and form amorphous or less orderly phase between spheroids. So, construction of defect material takes place which means the beginning of destruction.

It has been mentioned above, that under the influence of aggressive chemical mediums on polymers the phenomenon of chemical destruction takes place. The analysis of the research shows that in accordance with correlation of diffusion speeds of aggressive mediums and chemical reaction in polymer destruction speeds in aggressive mediums can take place in through following areas: inner diffusion - kinetic (destruction takes place in reaction zone, its size is increasing in time, and later it is spread on the whole volume of polymer material); inner kinetic area (destruction takes place with similar speed in accessible volume of polymer product ); outside diffusion - kinetic area (destruction takes place in thin surface layers of constant size, which can be monolayer of polymer in the limit ).

When aggressive mediums influence the polymeric compositional materials begins from the division surface "polymer - filler" as a result of weakening their adhesion properties, weakening, breaking the ties between them. Aggressive medium can contribute the washing away polymer binding agent. Both processes lead to the structure of compositional material destruction. To example the influence of parameters of processing of polymer materials by active environments on its activity under mechanism load some experimental research was done to example some samples under stretching after corresponding chemical processing. Some reactoplasts and thermoplastics were chosen, details and products of which are widely used in different branches of industry, among them: carpolon, getinaks, teksstolit. The samples were tested under constant conditions of experiment: the technology of producing samples, temperature, speed and quality of loading under monoaxonic stretching, according to GOST $11262-80$. The samples were preliminary processed by physically active medium toluol (for 5 and 15 hours ),room temperature. Then the samples underwent stretching, the speed of moving of loading equipment clamps was $12000 \mathrm{~mm} / \mathrm{hours}(0.33 \mathrm{~mm} / \mathrm{second})$ and was constant. 
It has been mentioned above, that under the influence of aggressive chemical mediums on polymers the phenomenon of chemical destruction takes place. The analysis of the research shows that in accordance with correlation of diffusion speeds of aggressive mediums and chemical reaction in polymer destruction speeds in aggressive mediums can take place in through following areas: inner diffusion - kinetic (destruction takes place in reaction zone, its size is increasing in time, and later it is spread on the whole volume of polymer material); inner kinetic area (destruction takes place with similar speed in accessible volume of polymer product); outside diffusion - kinetic area (destruction takes place in thin surface layers of constant size, which can be monolayer of polymer in the limit).

The analysis of the result shows that processing of examined polymer materials (caprolon, getinaks, textolit ) in active mediums lead to sufficient lowering of durability. It is proved by decreasing quality of necessary elasticity limit $\sigma_{m}$ for caprolon and quantity of broken tension $\sigma_{p}$ for textolit and getinaks. For example, when caprolon is processed by toluolom, the quantity $\sigma_{m}$ is decreasing from $85 \mathrm{MPa}$ up to $62 \mathrm{MPa}$ ( 5 hours in medium) and up to $54 \mathrm{MPa}$ (being in the medium for 15 hours ). For getinaks the lowering of the quantity $\sigma_{p}-$ from $118 \mathrm{MPa}$ up to $40 \mathrm{MPa}$, for textolit - from $102 \mathrm{MPa}$ up to 53 or $48 \mathrm{MPa}$ (being in the medium correspondingly 5 or 15 hours ).

The analysis of the results obtained (Figure 1-3)shows, that when the processing is done in the active medium, time of destruction for reactive polymer materials (gentinaks, textolit)is shortened and the shape of destruction curves of thermoplastic material is changed, the curves become sloping, without sharp extremum. These peculiarities of reactoplasts (getinaks and textolit ) activity can be explained by the described above processes which cause the change of material structure accompanied by lowering its durability. The changing of stretch curves of caprolon can be explained by decreasing of flexibility of polymer chainsaw of the material because of formation of conglomerations in active mediums. The conglomeration influence the destruction process because it proceeds in "weak place" in polymer structure (on conglomeration ties).

In cyclic deformations of polymer materials as well as in cases of contacts with moving solid surfaces multiple superposition o tension takes place, it seems to specify destruction regularities. But the regularities characterizing these processes in aggressive environments have not been researched yet. There are some results for rubbers, because they are widely used in products which undergo cyclic deformations in aggressive mediums.

To sum up, it can be said, that in the aspect of mechanical processing of polymer work pieces taking into consideration specific interruption of polymer materials with different aggressive environments it is necessary to hold preliminary chemical processing of work pieces (made from polymer materials ) to make the following processing by cutting more effective.

Due to organized making micro defects and due to their constant geometric parameters chemical agents are distributed along section of work piece every during the following chemical processing. Intensive inner corroding, deepening and widening the structure defects take place; it caused fragility of surface layer to a certain degree. The duration of chemical processing depends on time necessary for structural transformation of the material. In practice this parameter can be valued according to the following dependences for the first and second offered methods correspondingly:

$$
t=\frac{p \delta}{V}, t=\frac{p \delta}{V} \mathrm{~K}
$$


( $\mathrm{t}$ - time of processing, $\mathrm{p}$ - density of work piece material $\mathrm{kg} / \mathrm{m}^{3} ; \delta$ - thickness of cut allowance in one cycle, $\mathrm{V}$ - speed of dissolution of e component material in dissolvent, determined by experiment, K-coefficient of solidarity of work piece surface).

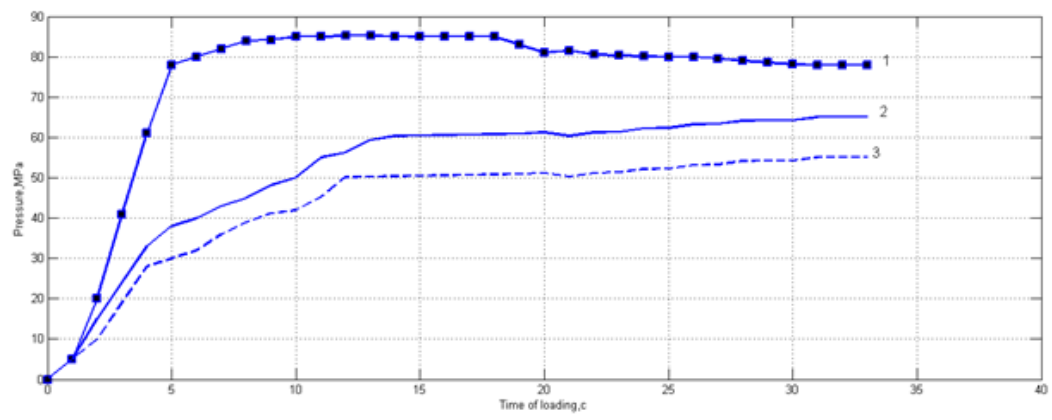

Figure 1. Diagram of Stretching Caprolon Samples after their Processing with Toulom: without Chemical Processing; 2-time of Processing (5 hours); 3-time of Processing (15 hours)

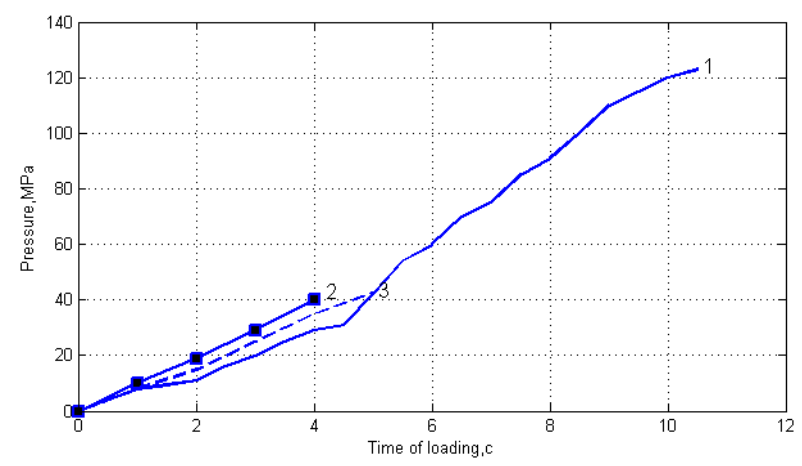

Figure 2. Diagram of Stretching Getinaks Samples after Processing with Toulom: 1-without Chemical Processing; 2-time of Processing (15 hours); 3Time of Processing (5 hours)

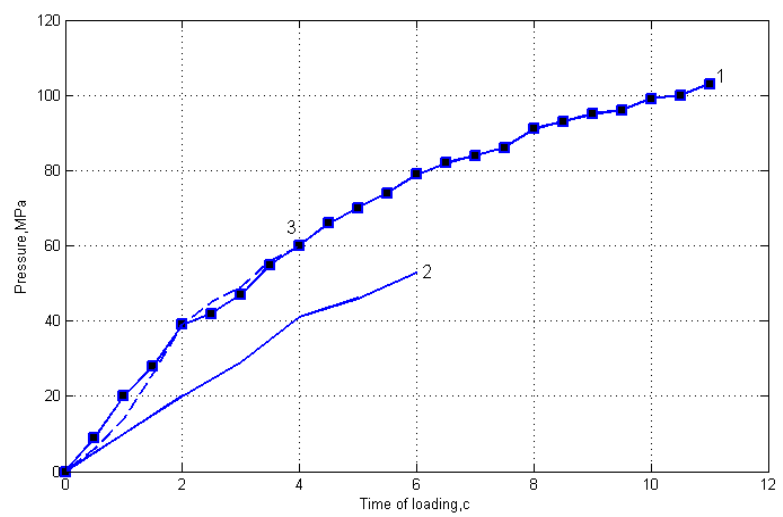

Figure 3. Diagram of Stretching Textolit Samples after Processing with Toulom: 1-without Chemical Processing; 2-time of Processing (15 hours); 3Time of Processing (5 hours) 
The process of mechanic processing by cutting polymer materials can be regarded as a kind of destruction process under influence of the main crack with formation of two surfaces - processed surface by cutting and outside surface. It is possible, that adsorption SAS, reducing the work, necessary to from new surface, lowers resistance to cutting and contributes to loosening the surface layers. Zone of pre-destruction is formed, it makes deformation process of cut layer easier and it is precondition for increasing the productivity of the process and quality of the surface processed by cutting.

\section{Conclusion}

The results of the experiments and conclusions have allowed to developing a method to process details made of plastic, the method has been patented. The essence of the method consists in directing change of properties of the processed material by preliminary processing of the surface layer of the work piece in the zone of cutting with its following interaction with cutting instrument. Processing of the component surface by chemical reagent the effects described above, and reduces the durability of the surface layer of the material. The structure of the material changes, chemical and intermolecular ties being described and the other part being stretches. So, the effect of "local fragility" of the part of the processed material surface takes place.

1. On the basis of the analysis of phenomenon, taking place when polymer material are in aggressive mediums and on the basis of experiments chemical influence on polymer workplaces before by cutting was proved. It is necessary to process polymers in aggressive medium in the way to ensure cracking or loosening surface layer to a certain depth without changeling chemical properties of a certain polymer.

2. Chemical - mechanical methods of processing polymer work pieces to ensure effectiveness of cutting process sing have been developed.

\section{Acknowledgements}

The work was supported by Jilin Provincial Science and Technology Department (No. 20140414025GH). The research activities have been funded by Changchun Science and Technology (No. 13GH15). The research activities also have been funded by Jilin Provincial Education Department (No. 2014290).

\section{References}

[1] H. Clements and H. Kestler, "Advanced Engineering Materials", vol. 2, (2000), p. 551.

[2] T. Tetsui, K. Shindo, S. Kobayashi and M. Takeyama, "Scripta Materialia", vol. 47, (2002), p. 399.

[3] K. P. Thurlow, "Electroless nickel plating on aluminum connectors [J]", Trans.Inst.(1989), pp. 67-82.

[4] Y. S. Zuyev, "Polymer destruction in aggressive mediums", - M.: Chemistry, (19720, p. 279.

[5] H. Qin, H. Li and X. Wang, "Hardware-software complex of transfer analysis features of NDT objects", ICMECE 2012, Applied Mechanics and Materials2013,V313-314: 1311-1315.

[6] H. Qin, N. Lia nd C. Li, "Spectral Analysis of Acoustic Emission Signals Using NI Systems", HSME 2012, Advanced Materials Research 2013,vol. 663, pp. 507-510.

[7] H. Qin, X.Wang and Q. Du, "Research of special form for analysis properties in transmit channels of NDT system", ICMECE 2012, Applied Mechanics and Materials, vol. 313-314, (2013), pp.1316-1319.

[8] H. Qin, G. Zhao, Q. Fan, T. Jia and H. Du, "The Nondestructive Testing Approach of Acoustic Emission for Environmentally Hazardous Objects", AEIT, (2013), pp. 474-477.

[9] H. W. Kerr and W. Kurz, "Solidification of peritectic alloys", International Materials reviews, vol. 41, no. 4, (1996), pp. 129-164.

[10] A. Fujishima and K. Honda, "Titanium dioxide photocatalysis [J]”, Nature, vol. 37, no. 1, (1972), pp. 238245. 
International Journal of Control and Automation Vol.8, No.3 (2015) 